\title{
El papel moderador de los estilos educativos parentales en la relación entre la personalidad y el consumo de cannabis
}

\author{
ANDREA CuEVAS CARDA \\ andrea.cuevas@uji.es \\ LAURA MEZQUITA GUILLAMÓN \\ Imezquit@uji.es
}

\section{Resumen}

Introducción: Estudios sobre personalidad indican que la apertura a la experiencia, la baja responsabilidad y la baja amabilidad son factores de riesgo para el consumo de cannabis. Además, variables ambientales como los estilos educativos parentales de escasa supervisión, excesiva permisividad y castigo se han relacionado también con un mayor consumo. No obstante, el efecto sinérgico entre ambas variables ha sido poco estudiado. Por ello, nuestro objetivo fue explorar en qué medida los estilos educativos parentales moderan la relación entre la personalidad y el consumo de cannabis. Método: 495 universitarios (media edad $=21,35(\mathrm{SD}=2,515), 64,6 \%$ mujeres) completaron el cuestionario de personalidad NEO PI-R, la escala sobre el consumo de cannabis CODIS, y el cuestionario de estilos educativos parentales APQ. Se realizaron análisis de regresión, siendo las variables dependientes la cantidad y la frecuencia de consumo de cannabis semanal, y las variables predictoras las dimensiones de personalidad, los estilos educativos parentales y sus interacciones. Para interpretar las interacciones significativas se realizaron gráficos de interacción y análisis de pendiente simple. Resultados: La baja responsabilidad, el castigo y la interacción entre ambas variables, así como la extraversión, la escasa supervisión y su interacción, predijeron una mayor frecuencia y cantidad de consumo de cannabis durante la semana. Discusión: Los resultados muestran que además de los efectos simples de la personalidad y los estilos educativos parentales, presentar ambas variables de riesgo (i.e., baja responsabilidad y castigo, extraversión y escasa supervisión) multiplicaría la probabilidad de consumir cannabis en los adultos jóvenes.

Palabras clave: personalidad, estilos educativos, cannabis, castigo, escasa supervisión.

\section{Abstract}

Introduction: Personality research suggests that openness to the experience, low conscientiousness and low agreeableness are risk factors for cannabis use. In addition, environmental variables such as parenting styles of low supervision, high permissiveness and punishment have also been related to higher cannabis consumption. 
However, the synergistic effect between these two variables has been less studied. Therefore, our objective was to explore the extent to which parenting styles moderate the relationship between personality and cannabis use. Method: Four hundred ninety five university students (mean age $=21,35(\mathrm{SD}=2,515), 64,6 \%$ women) completed the personality questionnaire NEO PI-R, the scale of cannabis CODIS, and the parenting questionnaire APQ. Regression analyzes were performed, the weekly quantity and frequency of cannabis were the dependent variables, and personality dimensions, parenting styles and their interactions were the independent variables. To interpret significant interactions, we performed interaction graphics and simple slope analysis.

Results: Low conscientiousness, punishment and the interaction between variables, and extraversion, low supervision and their interaction, predicted higher smoking frequency and quantity during the week. Discussion: The results show that in addition to the simple effects of personality and parenting styles, presenting both risk variables (i.e., low conscientiousness and punishment, extraversion and poor supervision) multiply the probability of consuming cannabis in young adults.

Keywords: personality, educational styles, cannabis, punishment, low supervision.

\section{Introducción}

El cannabis es la sustancia ilícita más consumida en el mundo según la United Nations Office on Drugs and Crime (UNODC, 2014).

En Europa, su consumo y accesibilidad ha aumentado, siendo España uno de los países donde más resina de cannabis se ha incautado. Además, es la tercera droga más consumida por detrás del alcohol y el tabaco, y está especialmente extendida entre los jóvenes-adultos españoles, un $38,4 \%$ de ellos se considera consumidor habitual de cannabis (Observatorio Europeo de las Drogas y las Toxicomanías (OEd. t.), 2012).

Existen múltiples variables biopsicosociales que pueden influir en el consumo de sustancias (Engel, 1977). Entre ellas, la personalidad y los estilos educativos parentales actuarían como factores distales e inespecíficos de vulnerabilidad al consumo de cannabis.

En cuanto a la relación de la personalidad y el consumo de cannabis, existe evidencia de que la apertura a la experiencia se asocia con mayores síntomas del consumo y dependencia (Flory, Lynam, Milich, Leukefeld y Clayton, 2002). Otros estudios realizados con el modelo de los cinco grandes encuentran que, además de las mayores puntuaciones en apertura a la experiencia, los consumidores de cannabis puntúan más bajo en amabilidad y responsabilidad que los no consumidores (Fridberg, Vollmer, O’Donnell, y Skosnik, 2011; Terracciano, Löckenhoff, Crum, Bienvenu, y Costa, 2008).

Los estudios de meta-análisis parecen corroborar la relación del consumo de cannabis con la alta apertura a la experiencia y la baja responsabilidad (Bogg y Roberts, 2004; Gorman y Derzon, 2002), mientras que el neuroticismo se asociaría con el consumo en muestras clínicas, una vez el patrón de uso de la sustancia fuese más problemático (Kotov, Gamez, Schmidt, y Watson, 2010).

Finalmente, el papel de la extraversión estaría menos claro. Así, mientras se ha hipotetizado que la extraversión se asociaría con un mayor consumo de cannabis (Terracciano y cols., 2008), estudios con adolescentes muestran asociaciones negativas entre ambas variables (Flory y cols., 2002). 
En lo que respecta a la influencia de los estilos educativos parentales en el consumo de cannabis, se ha hipotetizado que una excesiva permisividad por parte de los padres hacia el consumo, la presencia de castigos aplicados inadecuadamente o de forma excesiva o la falta de supervisión por parte de los padres favorecerían el consumo. Por otra parte, la percepción de control parental ha demostrado prevenir el consumo de alcohol, tabaco y cannabis, y a su vez retrasar la edad de inicio en el consumo. Del mismo modo, también un clima de apoyo familiar y modelos adecuados de consumo protegen ante el consumo de drogas en general (Becoña y cols., 2013). En definitiva, los estudios indican la importancia de un balance adecuado entre control y afecto, es decir, las características fundamentales del estilo democrático, a la hora de prevenir el consumo de drogas (Aleixandre y Navarro, 2008).

Existen pocos estudios que aborden la interrelación de la personalidad y los estilos educativos a la hora de predecir el consumo. Así, Creemers y cols. (2015) encontraron que aqueIlos adolescentes con altos niveles de neuroticismo, unido a un descenso en el control percibido, eran más vulnerables a iniciarse tempranamente en el consumo de cannabis. Del mismo modo, la introversión unida al descenso del control percibido también conducía a un inicio temprano en el consumo de cannabis. No obstante, esta asociación fue en contra de sus hipótesis iniciales, ya que era la alta extraversión la que se esperaba que se relacionase con el consumo (Creemers y cols., 2015).

Por otra parte, conociendo la convergencia entre los distintos factores de riesgo para el consumo de un gran número de drogas, no es de extrañar que, los mismos estilos educativos parentales, además de para el alcohol, pudiesen ser un factor de riesgo para el cannabis. En esta línea, Mezquita y cols. (2006) encontraron que la baja responsabilidad del adolescente unido a un estilo parental excesivamente permisivo o en el que predominaba el castigo se asociaba con un mayor consumo de alcohol (Mezquita y cols., 2006).

Sin embargo, la asociación entre el estilo educativo de los padres, los rasgos de personalidad del hijo y la aparición del consumo temprano de cannabis se ha explorado poco, principalmente en adolescentes y sigue siendo bastante desconocida en muestras de mayor edad.

Por tanto, el objetivo de la presente investigación fue estudiar la relación entre las dimensiones de personalidad, los estilos educativos y las interacciones entre ambas variables a la hora de predecir el consumo de cannabis en los adultos jóvenes.

Se hipotetizó que la baja responsabilidad y la apertura a la experiencia se relacionarían con un mayor de cannabis; por otra parte, también se hipotetizó que la baja responsabilidad, el neuroticismo o la extraversión en interacción con la escasa supervisión, el castigo o la inconsistencia disciplinaria se asociarían con un mayor consumo de cannabis.

\section{Método}

\section{Participantes}

La muestra está compuesta por 495 estudiantes de la Universitat Jaume I de Castellón (64,6 \% eran mujeres), con una media de edad de 21,35 años $(S D=2,515)$. El rango de edad de la muestra va de los 18 a los 45 años.

\section{Procedimiento}

La muestra se obtuvo durante 2011 y 2013 en la Universitat Jaume I. Los participantes fueron informados del proyecto, de la confidencialidad con la que se tratarían sus datos y de 
la posibilidad de abandonar la investigación en el momento que quisiesen. Parte de los instrumentos los completaron online, mientras que el cuestionario de consumo de sustancias lo completaron en el laboratorio. Todos ellos recibieron compensación económica por su participación.

\section{Instrumentos}

EI NEO-PI-R (Costa y McCrae, 1999) es un cuestionario que contiene 240 ítems, que a través de en una escala Likert de 5 puntos $(0=$ totalmente en desacuerdo a $5=$ totalmente de acuerdo) evalúa las cinco dimensiones de personalidad basadas en los cinco grandes: neuroticismo $(\mathrm{N})$, extraversión $(\mathrm{E})$, apertura a la experiencia $(\mathrm{O})$, amabilidad $(A)$ y responsabilidad (C).

El Cannabis and Other Drugs Intake Scale (CODIS) es un instrumento diseñado por el grupo de investigación para evaluar el consumo de cannabis y otras drogas de uno mismo, del mejor amigo, del grupo de amigos, de la pareja y de la familia. En el presente estudio se utilizaron las escalas de frecuencia $(0=$ ningún día de la semana a $7=$ todos los días de la semana) y cantidad semanal ( $n^{\circ}$ de porros) del consumo propio de cannabis.

Alabama Parenting Questionnaire (APQ; Shelton, Frick y Wootton, 1996). Este cuestionario evalúa estilos educativos parentales a través de 42 ítems a través de una escala Likert de 5 puntos $(1=$ nunca a $5=$ siempre $)$. Consta de 5 subescalas: implicación, estilo educativo positivo, escasa supervisión, inconsistencia en la disciplina y castigo físico.

\section{Análisis}

Mediante el uso del software sPSS versión 21, se realizaron análisis descriptivos de la muestra total, para hombres y para mujeres; posteriormente se calcularon las diferencias de sexo mediante una prueba $t$ y también el tamaño del efecto de estas diferencias a través de la $d$ de Cohen (1992), mediante la calculadora online del tamaño del efecto (Becker, n.d.).

Mediante este mismo software, se realizaron análisis de regresión lineal simple, siendo las variables dependientes la cantidad y frecuencia de consumo total de cannabis a la semana. Tras controlar el efecto de la edad y el género, se introdujeron como variables predictoras las dimensiones de personalidad, el castigo o la escasa supervisión y las interacciones entre ambas. Una vez realizado el análisis, para interpretar las interacciones significativas se utilizó un análisis de pendiente simple y la representación gráfica de la interacción (Dawson, 2014).

\section{Resultados}

\section{Análisis descriptivos}

Los análisis descriptivos aparecen en la tabla 1. Las mujeres puntuaron significativamente más alto en neuroticismo y amabilidad; mientras que los hombres reciben menos supervisión que las mujeres, y su frecuencia y cantidad de consumo de cannabis semanal fue mayor. 


\section{Análisis de regresión}

Los análisis de regresión mostraron que la responsabilidad se asoció negativamente con ambas variables de consumo de cannabis (véase tabla 2). Además la responsabilidad interaccionó con el castigo a la hora de predecir tanto la cantidad como la frecuencia de consumo semanal (véase tabla 2 y figura 1). Por otra parte, también se observaron efectos de interacción significativos entre extraversión y escasa supervisión tanto en la cantidad como en la frecuencia de consumo total de cannabis semanal (véase tabla 2). Estos efectos de interacción se representaron gráficamente en la figura 2 .

Tabla 1

Análisis descriptivos de la muestra total y diferenciando por sexo

\begin{tabular}{lccccc}
\hline & $\begin{array}{c}\text { Muestra total } \\
(\mathrm{N}=495) \\
\mathrm{M}(\mathrm{SD})\end{array}$ & $\begin{array}{c}\text { Hombres } \\
(\mathrm{N}=179) \\
\mathrm{M}(\mathrm{SD})\end{array}$ & $\begin{array}{c}\text { Mujeres } \\
(\mathrm{N}=313) \\
\mathrm{M}(\mathrm{SD})\end{array}$ & $t$ & $d$ \\
\hline Personalidad & & & & \\
$\mathrm{N}$ & $92,27(21,08)$ & $85,90(20,33)$ & $95,80(20,69)$ & $-4,99^{* * *}$ & 0,48 \\
$\mathrm{E}$ & $115,47(17,50)$ & $117,10(17,80)$ & $114,57(17,29)$ & 1,51 & 0,14 \\
O & $114,33(17,26)$ & $111,94(17,34)$ & $115,66(17,10)$ & $-2,24^{*}$ & 0,22 \\
A & $115,22(16,28)$ & $107,74(15,75)$ & $119,36(15,07)$ & $-7,87^{* * *}$ & 0,75 \\
C & $112,14(20,49)$ & $111,46(18,31)$ & $112,52(21,18)$ & $-0,55$ & 0,05 \\
\hline Castigo & $2,23(2,31)$ & $2,42(2,26)$ & $2,12(2,33)$ & 1,25 & 0,13 \\
\hline $\begin{array}{l}\text { Escasa } \\
\text { supervisión }\end{array}$ & $8,66(5,94)$ & $10,54(6,08)$ & $7,65(5,61)$ & $4,85^{* * *}$ & 0,49 \\
\hline $\begin{array}{l}\text { Frecuencia total } \\
\text { consumo }\end{array}$ & $0,98(2,43)$ & $1,59(3,25)$ & $0,65(1,77)$ & $4,00^{* * *}$ & 0,36 \\
\hline $\begin{array}{l}\text { Cantidad total } \\
\text { consumo }\end{array}$ & $1,53(4,91)$ & $2,80(7,14)$ & $0,82(2,81)$ & $4,19 *$ *** & 0,36 \\
\hline
\end{tabular}

Nota: Los valores de la $d$ de Cohen $0,20,0,50$ y 0,80 corresponden a efectos pequeños, medianos y grandes respectivamente (Cohen, 1992). ${ }^{*} p<0,05,{ }^{* *} p<0,01,{ }^{* * *} p<0,001$. 
Tabla 2

Análisis de regresión

\begin{tabular}{|c|c|c|c|c|c|c|c|c|c|}
\hline \multirow[b]{3}{*}{$\begin{array}{c}\text { Variables } \\
\text { independientes }\end{array}$} & \multicolumn{4}{|c|}{ Variables dependientes } & & \multicolumn{4}{|c|}{ Variables dependientes } \\
\hline & \multicolumn{2}{|c|}{$\begin{array}{c}\text { Frecuencia } \\
\text { de consumo total }\end{array}$} & \multicolumn{2}{|c|}{$\begin{array}{c}\text { Cantidad } \\
\text { de consumo total }\end{array}$} & \multirow[b]{2}{*}{$\begin{array}{c}\text { Variables } \\
\text { independientes }\end{array}$} & \multicolumn{2}{|c|}{$\begin{array}{c}\text { Frecuencia } \\
\text { de consumo total }\end{array}$} & \multicolumn{2}{|c|}{$\begin{array}{c}\text { Cantidad } \\
\text { de consumo total }\end{array}$} \\
\hline & $\beta$ & $\Delta \mathrm{R}^{2}$ & $\beta$ & $\Delta R^{2}$ & & $\beta$ & $\Delta R^{2}$ & $\beta$ & $\Delta R^{2}$ \\
\hline Género & $-0,16^{\star *}$ & $0,03^{* *}$ & $-0,17^{\star *}$ & $0,03^{* *}$ & Género & $-0,16^{\star *}$ & $0,03^{* *}$ & $-0,16^{\star \star}$ & $0,03^{* *}$ \\
\hline Edad & $-0,06$ & & $-0,05$ & & Edad & $-0,06$ & & $-0,02$ & \\
\hline$N$ & $-0,01$ & $0,06^{\star *}$ & $-0,01$ & $0,05^{\star *}$ & $N$ & $-0,03$ & $0,06^{* *}$ & $-0,03$ & $0,06^{* *}$ \\
\hline$E$ & 0,10 & & 0,09 & & E & 0,09 & & 0,08 & \\
\hline $\mathrm{O}$ & 0,03 & & 0,05 & & $\mathrm{O}$ & 0,04 & & 0,05 & \\
\hline A & 0,03 & & 0,02 & & A & 0,01 & & 0,01 & \\
\hline C & $-0,15^{\star *}$ & & $-0,14^{*}$ & & C & $-0,15^{\star *}$ & & $-0,14^{*}$ & \\
\hline Castigo & 0,08 & & 0,07 & & $\begin{array}{l}\text { Escasa } \\
\text { Supervisión }\end{array}$ & 0,04 & & 0,03 & \\
\hline C x Castigo & $-0,12^{*}$ & & $-0,12^{*}$ & & $\begin{array}{l}\text { E x Escasa } \\
\text { supervisión }\end{array}$ & $-0,17^{*}$ & & $-0,18^{* *}$ & \\
\hline
\end{tabular}

\section{Cantidad consumo cannabis}

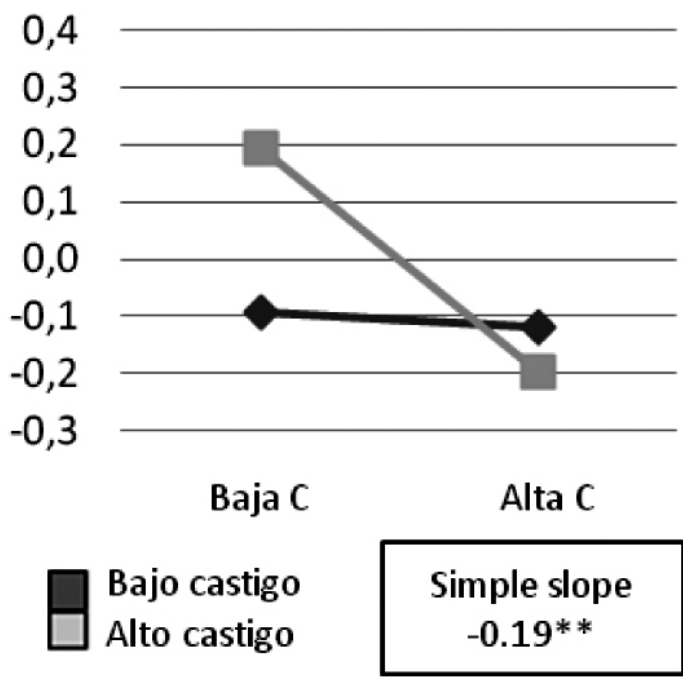

Frecuencia consumo cannabis

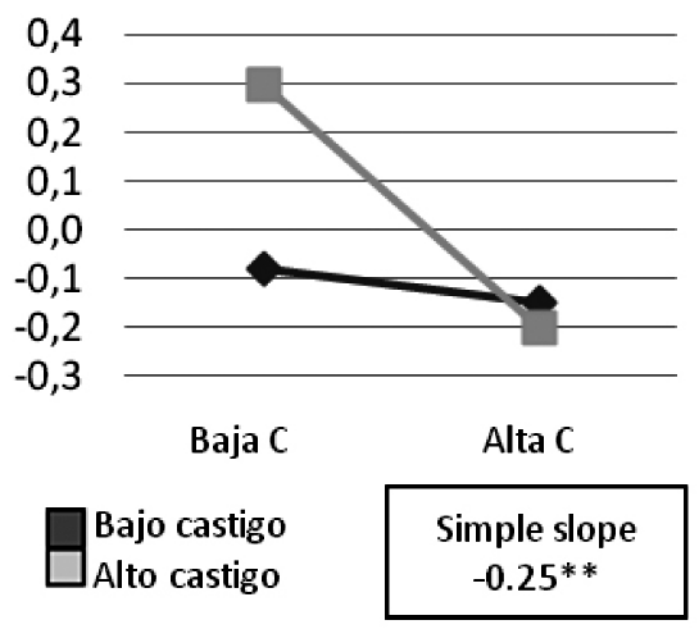

Figura 1. Gráfica de interacción responsabilidad x castigo a la hora de predecir el consumo de cannabis 


\section{Cantidad consumo cannabis}

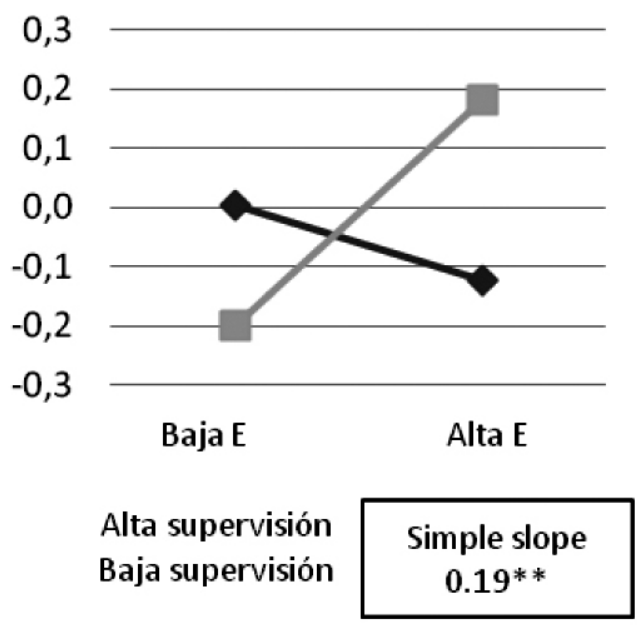

\section{Frecuencia consumo} cannabis

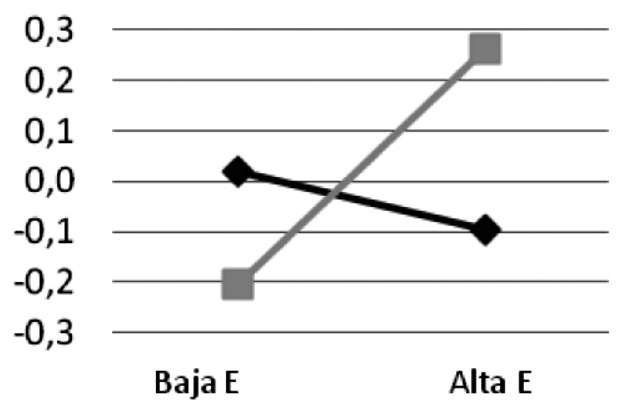

Alta supervisión Baja supervisión
Simple slope

$0.23^{* *}$

Figura 2. Gráfica de interacción extraversión x baja supervisión a la hora de predecir el consumo de cannabis

\section{Discusión}

El objetivo de la presente investigación fue estudiar la relación entre las dimensiones de personalidad, los estilos educativos y las interacciones entre ambas variables a la hora de predecir el consumo de cannabis en los adultos jóvenes.

En lo referente a los efectos directos, en el presente estudio, del mismo modo que en estudios previos, se ha encontrado que la baja responsabilidad se asocia con un mayor consumo de cannabis (Fridberg y cols., 2011; Terracciano y cols., 2008). No obstante, no se han obtenido asociaciones significativas entre la apertura a la experiencia y el consumo semanal de cannabis. Una posible explicación ante este hecho es que las asociaciones no significativas entre la apertura a la experiencia y el consumo podrían deberse a las características de la muestra (i.e., $75,2 \%$ no ha consumido nunca).

En cuanto a los estilos educativos parentales, en el presente estudio no se encuentra relación directa entre estilos educativos y consumo de cannabis aunque sí ejercen un papel moderador en la relación de personalidad y consumo. No obstante, la evidencia previa indica que los estilos educativos afectan al inicio del consumo y al consumo de adolescentes (Becoña y cols., 2013), sin embargo hay menos estudios con jóvenes y adultos. Otro punto importante es que en esta muestra los estilos educativos son retrospectivos, es decir, de la infancia, y aun así encontramos relación con el consumo en la edad adulta.

Por lo que respecta a los efectos de interacción entre personalidad y estilos educativos parentales, en este estudio, siguiendo la línea de los estudios previos realizados con consumo de alcohol en adolescentes (Mezquita y cols., 2006); encontramos que aquellos jóvenes que presentan baja responsabilidad y que además recibieron un estilo educativo basado en el castigo por parte de sus padres, tienden a consumir más cannabis.

La extraversión también se relacionó de forma positiva con el consumo de cannabis en el presente estudio. Al parecer, niveles altos de extraversión favorecen el consumo de cannabis en aquellos jóvenes que experimentan escasa supervisión parental. Esto podría ser debido a que se favorece que encuentren más situaciones para el consumo. Estos resultados van en la línea de lo esperado en estudios previos realizados con adolescentes (Creemers y cols., 
2015). Sin embargo, sigue sin estar totalmente claro cuál es el papel que ejerce exactamente la extraversión en la relación entre estilos educativos y consumo de cannabis.

En contra de lo esperado, en el presente estudio, el neuroticismo no se relaciona significativamente con estilos educativos y consumo. Posiblemente esto sea debido a las características de la muestra, ya que los que consumían lo hacían de forma ocasional y, recordemos que es el abuso y dependencia del cannabis y otras drogas en general lo que se ha asociado con neuroticismo en estudios previos (Bogg y Roberts, 2004; Kotov, Gamez, Schmidt, y Watson, 2010). Por tanto, estos resultados van en contra de los hallados en estudios previos, en los que el neuroticismo unido a un descenso en el control parental aumentaba la vulnerabilidad ante el consumo de cannabis (Creemers y cols., 2015).

Finalmente, se desprende que el efecto de las características de personalidad de riesgo para el consumo de cannabis se ve exacerbado ante la existencia de estilos educativos de riesgo. Concretamente, en este estudio se ha encontrado significativa la relación entre extraversión y escasa supervisión; y responsabilidad, con castigo.

\section{Referencias bibliográficas}

Aleixandre, N. L., y Navarro, S. T. (2008). Influencia de los estilos educativos paternos en el consumo de drogas en adolescentes. Originales, 33, 288-299.

Becker. L. (n.d). Effect size calculator. Recuperado el 23 de mayo de 2015, de http://www.uccs. edu/ Ibecker/.

Becoña, E., Martínez, Ú., Calafat, A., Fernández-Hermida, J. R., Juan, M., Sumnall, H., y Gabrhelík, R. (2013). Parental permissiveness, control, and affect and drug use among adolescents. Psicothema, 25, 292-298.

Bogg, T., y Roberts, B. W. (2004). Conscientiousness and health-related behaviors: a meta analysis of the leading behavioral contributors to mortality. Psychological Bulletin, 130, 887-919.

Cohen, J. (1992). A power primer. Psychological Bulletin, 112, 155-159.

Cooper, M. L., Agocha, V. B., y Sheldon, M. S. (2000). A motivational perspective on risky behaviors: The role of personality and affect regulatory processes. Journal of Personality, 68, 1059-1088.

Costa, P. T., y McCrae, R. R. (1999). Revised NEO Personality Inventory (NEO-PI-R) and NEO Five-Factor Inventory (NEO-FFI). Professional manual. Madrid: TEA.

Creemers, H. E., Buil, J. M., van Lier, P. a. C., Keijsers, L., Meeus, W., Koot, H. M., y Huizink, A. C. (2015). Early onset of cannabis use: Does personality modify the relation with changes in perceived parental involvement? Drug and Alcohol Dependence, 146, 61-67.

Dawson, J. F. (2014). Moderation in management research: What, why, when, and how. Journal of Business and Psychology, 29, 1-19.

Engel, G. (1977). The need for a new medical model: a challenge for biomedicine. Science, 196, 129-136.

Flory, K., Lynam, D., Milich, R., Leukefeld, C., y Clayton, R. (2002). The relations among personality, symptoms of alcohol and marijuana abuse, and symptoms of comorbid psychopathology: Results from a community sample. Experimental and Clinical Psychopharmacology, 10, 425-434.

Fridberg, D. J., Vollmer, J. M., O’Donnell, B. F., y Skosnik, P. D. (2011). Cannabis users differ from non-users on measures of personality and schizotypy. Psychiatry Research, 186, 46-52. 
Gorman, D. M., y Derzon, J. H. (2002). Behavioral traits and marijuana use and abuse: a metaanalysis of longitudinal studies. Addictive Behaviors, 27, 193-206.

Kotov, R., Gamez, W., Schmidt, F., y Watson, D. (2010). Linking «big» personality traits to anxiety, depressive, and substance use disorders: a meta-analysis. Psychological Bulletin, $136,768-821$.

Mezquita, L., Moya, J., Edo, S., Maestre, E., Viñas, M., Ortet, G y Ibáñez, M. I. (2006). Personalidad y estilos educativos parentales como consumo de alcohol. Forum de Recerca, 12, 1-13.

Oedt (2012). Informe nacional 2012 (datos del 2011). Madrid: Reitox.

Shelton, K. K., Frick, P. J., y Wootton, J. (1996). The assessment of parenting practices in families of elementary school-aged children. Journal of Clinical Child Psychology, 25, 317327.

Terracciano, A., Löckenhoff, C. E., Crum, R. M., Bienvenu, O. J., y Costa, P. T. (2008). FiveFactor Model personality profiles of drug users. BMC Psychiatry, 8, 22.

United Nations Office on Drugs and Crime (UNODC). (2014). World Drug Report 2014. Viena: United Nations Publications. 